TRANSACTIONS OF THE

AMERICAN MATHEMATICAL SOCIETY

Volume 357, Number 8, Pages 3239-3253

S 0002-9947(05)03790-6

Article electronically published on January 27, 2005

\title{
THE SMOOTHING PROPERTY FOR A CLASS OF DOUBLY NONLINEAR PARABOLIC EQUATIONS
}

\author{
CARSTEN EBMEYER AND JOSÉ MIGUEL URBANO
}

\begin{abstract}
We consider a class of doubly nonlinear parabolic equations used in modeling free boundaries with a finite speed of propagation. We prove that nonnegative weak solutions satisfy a smoothing property; this is a well-known feature in some particular cases such as the porous medium equation or the parabolic $p$-Laplace equation. The result is obtained via regularization and a comparison theorem.
\end{abstract}

\section{INTRODUCTION}

This paper deals with a class of partial differential equations - doubly nonlinear parabolic equations - that have recently attracted a lot of attention. They arise in many different physical contexts such as, for instance, the description of turbulent filtration in porous media, or the flow of a gas through a porous medium in a turbulent regime; in general, doubly nonlinear parabolic equations are used to model processes obeying a nonlinear Darcy law (see 8, [14, and the references given therein).

Typical examples of such parabolic PDEs are equations of the form

$$
u_{t}=\Delta_{p}\left(|u|^{m-1} u\right), \quad m(p-1)>1,
$$

where $\Delta_{p}$ is the $p$-Laplacian, which are used in modeling phenomena involving a free boundary with a finite speed of propagation. These degenerate equations exhibiting a doubly nonlinearity generalize the porous medium equation $(p=2)$ and the parabolic $p$-Laplace equation $(m=1)$.

The aim of the paper is to show that nonnegative solutions of a class of doubly nonlinear parabolic equations satisfy the smoothing property, i.e., the estimate

$$
u_{t} \geq-\frac{c}{t} u
$$

where $c$ is a constant depending only on the data. The smoothing property (1.1) implies the regularizing property

$$
\left\|u_{t}\right\|_{L^{1}\left(\mathbb{R}^{d}\right)} \leq \frac{2 c}{t}\left\|u_{0}\right\|_{L^{1}\left(\mathbb{R}^{d}\right)},
$$

Received by the editors November 12, 2002 and, in revised form, November 19, 2003.

2000 Mathematics Subject Classification. Primary 35K65; Secondary 35R35, 76S05.

Key words and phrases. Degenerate parabolic equation, free boundary, finite speed of propagation, porous medium equation.

The second author was supported in part by the Project FCT-POCTI/34471/MAT/2000 and CMUC/FCT. 
as will be shown below, and plays a crucial role in the study of the finite speed of propagation of the free boundary (see, e.g., [5, 12, where the porous medium equation is treated) or the proof of regularity results for the solutions (cf. [13]).

The smoothing property is known for some particular degenerate equations although a systematic approach to the matter is still lacking. For instance, let $b$ be nondecreasing, $b(0)=0$, and

$$
c_{1} \leq \frac{b(s) b^{\prime \prime}(s)}{\left(b^{\prime}(s)\right)^{2}} \leq c_{2}
$$

for certain constants $c_{1}, c_{2}>0$. Then there is a constant $c$, depending only on $c_{1}$ and $c_{2}$, such that solutions of

$$
u_{t}-\Delta b(u)=0
$$

satisfy estimate (1.1) (cf. [6, 12]). A first proof of the smoothing property was given in 1 for the case of the porous medium equation

$$
u_{t}=\Delta u^{m} \quad(m>1) .
$$

In [17] the smoothing property was obtained for the one-dimensional porous medium equation with weak absorption

$$
u_{t}=\partial_{x} \partial_{x} u^{m}-c u^{k} \quad(c \geq 0, k \geq m) .
$$

The $n$-dimensional case is treated in 26]. Further proofs for other classes of porous medium type equations can be found in [6], 7], and [23]. The parabolic $p$-Laplace equation

$$
u_{t}=\operatorname{div}\left(|\nabla u|^{p-2} \nabla u\right)
$$

was considered in [15], with $p>2-\frac{2}{d+1}$. A proof of the smoothing property for solutions of the doubly nonlinear parabolic equation

$$
u_{t}=\partial_{x}\left(\left|\partial_{x} b(u)\right|^{p-2} \partial_{x} b(u)\right)
$$

in the one-dimensional case is given in [14].

The paper is organized as follows. The problem and the main result are stated in the next section. Then, in section 3, we introduce a regularized problem and study its most important properties. Section 4 contains the proof of the smoothing property as a consequence of a series of intermediate lemmas; essential use is made of the regularization and a comparison argument.

\section{The main Result}

Let $x \in \mathbb{R}^{d}, d \geq 2$, and $t \in(0, T]$, with $T<\infty$. We consider the Cauchy problem

$$
\left\{\begin{aligned}
\frac{\partial}{\partial t} u(x, t)=\sum_{i=1}^{d} \partial_{i} a_{i}(\nabla b(u(x, t)))-f(u(x, t)) & \text { in } \quad \mathbb{R}^{d} \times(0, T], \\
u(x, 0)=u_{0}(x) & \text { in } \quad \mathbb{R}^{d},
\end{aligned}\right.
$$

where

$$
\begin{array}{rlrl}
b(z) & =|z|^{m-1} z & & \text { for } z \in \mathbb{R}, \\
a_{i}(s) & =|s|^{p-2} s_{i} & \text { for } s \in \mathbb{R}^{d}, \quad i=1, \ldots, d, \\
f(z) & =|z|^{k-1} z & \text { for } z \in \mathbb{R} .
\end{array}
$$

We assume that $m>1, p>1$ and treat the slow diffusion case

$$
m(p-1)>1
$$


with weak absorption $k \geq m(p-1)$. Concerning the initial condition, we take $u_{0}(x) \geq 0$ and spt $u_{0}$ to be bounded. For simplicity, we restrict ourselves to $L^{\infty}$-initial data but $u_{0}$ can more generally be in $L^{1}$, in which case we have to approximate it with $L^{\infty}$-functions.

The pertinent definition of a weak solution for the problem is

Definition 2.1. We say that $u(x, t)$ is a weak solution of (2.1) if

$$
\begin{aligned}
u \in L^{\infty}\left(0, T ; L^{\infty}\left(\mathbb{R}^{d}\right)\right) ; \quad b(u) \in L^{p}\left(0, T ; W^{1, p}\left(\mathbb{R}^{d}\right)\right) & \\
-\int_{0}^{T} \int_{\mathbb{R}^{d}} u \phi_{t} \mathrm{~d} x \mathrm{~d} t & +\sum_{i=1}^{d} \int_{0}^{T} \int_{\mathbb{R}^{d}} a_{i}(\nabla b(u)) \partial_{i} \phi \mathrm{d} x \mathrm{~d} t \\
& +\int_{0}^{T} \int_{\mathbb{R}^{d}} f(u) \phi \mathrm{d} x \mathrm{~d} t=\int_{\mathbb{R}^{d}} u_{0} \phi(\cdot, 0) \mathrm{d} x,
\end{aligned}
$$

for all $\phi \in L^{p}\left(0, T ; W^{1, p}\left(\mathbb{R}^{d}\right)\right) \cap W^{1,1}\left(0, T ; L^{1}\left(\mathbb{R}^{d}\right)\right)$ such that $\phi(\cdot, T) \equiv 0$.

Remark 2.2. The existence of a weak solution may be proven as in [16], where doubly nonlinear parabolic equations are considered. Existence and uniqueness results concerning related equations are given in [3, 4, 20] and [19, 21], respectively.

Remark 2.3. It is well known that $u$ is Hölder continuous; see [9, 19, 22, 24, 25, 27].

Remark 2.4. As in [16] it can be shown that

$$
u_{t} \partial_{t} b(u) \in L^{1}\left(0, T ; L^{1}\left(\mathbb{R}^{d}\right)\right) .
$$

Since $u_{0} \geq 0$, the weak solution is nonnegative. In fact, applying a comparison theorem (see, e.g., [4, 18]) we find that $0 \leq u \leq\left\|u_{0}\right\|_{L^{\infty}}$. Moreover, spt $u(x, T)$ is bounded. To see this, let us consider the self-similar Barenblatt solutions (cf. [2])

$$
u^{*}(x, t ; \alpha, \tau)=(t+\tau)^{-\frac{1}{\mu}}\left[\left\{\alpha-k\left(|x|(t+\tau)^{-\frac{1}{n \mu}}\right)^{\frac{p}{p-1}}\right\}^{+}\right]^{\frac{p-1}{m(p-1)-1}},
$$

where $\mu=m(p-1)-1+\frac{p}{d}, k=\frac{m(p-1)-1}{m p}(d \mu)^{-\frac{1}{p-1}}$, and $\alpha, \tau>0$. Clearly, spt $u^{*}(x, T ; \alpha, \tau)$ is bounded for each $T>0$. Let $0 \leq u_{0} \leq u_{0}^{*}$ and $T>0$. From the comparison theorem it follows that $u \leq u^{*}$ on $\mathbb{R}^{d} \times[0, T]$. Thus,

$$
\text { spt } u(x, t) \subset \operatorname{spt} u^{*}(x, T ; \alpha, \tau) \quad \text { for all } t \in[0, T] .
$$

Further, it holds that

$$
\text { spt } u(\cdot, t) \subset \operatorname{spt} u(\cdot, s) \quad \text { for all } t<s .
$$

Now let us take a convex polyhedron $\Omega \subset \mathbb{R}^{d}$ such that spt $u(\cdot, T) \subset \subset \Omega$. It follows that

$$
\text { spt } u(\cdot, t) \subset \Omega \quad \text { for all } t \in[0, T]
$$

and, in fact, $u=0$ on $\partial \Omega \times(0, T]$. Hence, we may rewrite the Cauchy problem as

$$
\begin{array}{ll}
u_{t}=\sum_{i=1}^{d} \partial_{i} a_{i}(\nabla b(u))-f(u) & \text { in } \Omega \times(0, T], \\
u(x, t)=0 & \text { on } \partial \Omega \times(0, T], \\
u(x, 0)=u_{0}(x) & \text { in } \Omega .
\end{array}
$$


Our main result is as follows.

Theorem 2.5. Let $u$ be a nonnegative weak solution of (2.1) in the sense of the previous definition. Then

$$
u_{t} \geq-\frac{c}{t} u \quad \text { in } \mathcal{D}^{\prime}\left(\mathbb{R}^{d} \times(0, T)\right),
$$

where $c=\frac{1}{m(p-1)-1}$.

As a consequence we get

Corollary 2.6. Let $u$ be a nonnegative weak solution of (2.1) in the sense of the previous definition. For a.e. $t>0$, the following estimate holds:

$$
\int_{\mathbb{R}^{d}}\left|u_{t}(x, t)\right| d x \leq \frac{2 c}{t} \int_{\mathbb{R}^{d}}\left|u_{0}(x)\right| d x
$$

where $c=\frac{1}{m(p-1)-1}$.

\section{The REgularized PROBlem}

Now we define a regularization of problem 2.5) as follows. We consider the initial boundary value problem

$$
\begin{array}{ll}
u_{t}^{\varepsilon}=\sum_{i=1}^{d} \partial_{i} a_{i}\left(\nabla b\left(u^{\varepsilon}\right)\right)-f\left(u^{\varepsilon}\right) & \text { in } \Omega \times(0, T], \\
u^{\varepsilon}(x, t)=\varepsilon & \text { on } \partial \Omega \times(0, T], \\
u^{\varepsilon}(x, 0)=\bar{u}_{0}(x)+\varepsilon \equiv u_{0}^{\varepsilon} & \text { in } \Omega,
\end{array}
$$

where $\varepsilon \in(0,1)$ is small and $\bar{u}_{0}$ is a smooth (at least $C^{1}$ ) approximation of $u_{0}$, such that $u^{\varepsilon}(x, 0) \geq \varepsilon_{0}>0$ for some $\varepsilon_{0} \in(0, \varepsilon)$.

Remark 3.1. As before, it can be shown that there exists a unique weak solution $u^{\varepsilon}$. Furthermore, a comparison argument provides the inequality $u^{\varepsilon}(x, t) \geq \varepsilon_{0} e^{-t}$, valid for all $(x, t) \in \Omega \times(0, T]$. This implies that

$$
b^{\prime}\left(u^{\varepsilon}\right) \geq c_{0}>0
$$

for $c_{0}=m \varepsilon_{0}^{m-1} e^{-(m-1) T}$.

Multiplying the equation in (3.1) by a smooth test function $\phi$, such that $\phi(\cdot, T)=$ 0 , and integrating by parts, we obtain

$$
\begin{aligned}
-\int_{0}^{T} \int_{\Omega} u^{\varepsilon} \phi_{t} & +\sum_{i=1}^{d} \int_{0}^{T} \int_{\Omega}\left(b^{\prime}\left(u^{\varepsilon}\right)\right)^{p-1} a_{i}\left(\nabla u^{\varepsilon}\right) \partial_{i} \phi \\
& +\int_{0}^{T} \int_{\Omega} f\left(u^{\varepsilon}\right) \phi=\int_{\Omega} u_{0}^{\varepsilon} \phi(\cdot, 0) .
\end{aligned}
$$

In view of (3.2), the coefficient $\left(b^{\prime}\left(u^{\varepsilon}\right)\right)^{p-1}$ in (3.3) is bounded from below by a positive constant. Hence, in (3.3) there is no longer a double degeneracy.

A simple but crucial result is

Lemma 3.2. The solution $u^{\varepsilon}$ of (3.3) satisfies

$$
u_{t}^{\varepsilon} \in L^{2}\left(0, T ; L^{2}(\Omega)\right) \text {. }
$$


Proof. Due to (2.4) we have $u_{t}^{\varepsilon} \partial_{t} b\left(u^{\varepsilon}\right) \in L^{1}\left(0, T ; L^{1}(\Omega)\right)$. We conclude from inequality (3.2) that

which gives the result.

$$
\int_{0}^{T} \int_{\Omega} u_{t}^{\varepsilon} \partial_{t} b\left(u^{\varepsilon}\right) \geq c_{0} \int_{0}^{T} \int_{\Omega}\left(u_{t}^{\varepsilon}\right)^{2}
$$

We now approximate the regularized problem (3.1) using a Galerkin procedure. We consider a family of decompositions of $\Omega$ into closed $d$-simplices. We get a sequence of finite element spaces $V^{n}$ such that $V^{n} \subset V^{k}$ for $n \leq k$. Here, $V^{n}:=$ $\operatorname{span}\left\{\varphi_{1}, \ldots, \varphi_{n}\right\}$ is a space of continuous functions that are piecewise linear with respect to the triangulation of $\Omega$ and vanish on $\partial \Omega$.

Let $\Pi_{n} g(x)$ be the piecewise linear interpolant of a continuous function $g(x)$. That is, $\Pi_{n} g(x)$ is the continuous piecewise linear function satisfying $\Pi_{n} g\left(x_{j}\right)=$ $g\left(x_{j}\right)$ for all nodes $x_{j}$ of the triangulation of $\Omega$. Further, let us introduce the notation

and the spaces

$$
\left(g_{1}(x), g_{2}(x)\right)_{n}=\int_{\Omega} \Pi_{n}\left(g_{1}(x) g_{2}(x)\right) d x
$$

$$
S_{i}^{n}(\alpha)=\left\{f \in H^{i}\left(0, T ; L^{\infty}(\Omega)\right): f(x, t)=\alpha+\sum_{j=1}^{n} f_{j}^{n}(t) \varphi_{j}(x)\right\}, \quad i=0,1,
$$

given a constant $\alpha$ and functions $f_{j}^{n} \in H^{i}(0, T) \equiv W^{i, 2}(0, T)$. Observe that if $f \in S_{i}^{n}(\alpha)$, then $f=\alpha$ on $\partial \Omega \times(0, T)$.

The approximate problem consists in finding a function

$$
u^{n}(x, t)=b^{-1}\left(\varepsilon^{m}+\sum_{j=1}^{n} \gamma_{j}^{n}(t) \varphi_{j}(x)\right),
$$

with $\gamma_{j}^{n} \in L^{2}(0, T)$, such that

$$
\left(u_{t}^{n}, \phi^{n}\right)_{n}+\sum_{i=1}^{d} \int_{\Omega} a_{i}\left(\nabla b\left(u^{n}\right)\right) \partial_{i} \phi^{n} \mathrm{~d} x+\left(f\left(u^{n}\right), \phi^{n}\right)_{n}=0,
$$

for all test functions $\phi^{n} \in S_{0}^{n}(0)$, and

$$
u^{n}(0) \equiv u_{0}^{n}=b^{-1}\left(\Pi_{n} b\left(u_{0}^{\varepsilon}\right)\right) .
$$

Here, $\Pi_{n} b\left(u_{0}^{\varepsilon}\right)$ is the interpolant of $b\left(u_{0}^{\varepsilon}\right)$.

It follows from classical results that there exists a unique weak solution $u^{n}$. Let us state some regularity results for $u^{n}$. As above (cf. (2.4) and (3.10)) it can be shown that $\gamma_{j}^{n} \in H^{1}(0, T)$. Due to the structure of $u^{n}$ this implies that

$$
\nabla \partial_{t} b\left(u^{n}\right) \in L^{2}\left(0, T ; L^{2}(\Omega)\right) .
$$

Moreover, $u^{n}$ is an $L^{\infty}$-function, which follows from a comparison argument similar to the one used in Lemma 3.3 below. Due to the special structure of (3.6), the usual conditions on the acuteness of the triangulation (see [11]) are not needed in order to prove this semi-discrete maximum principle.

Noting that $V^{n} \subset W_{0}^{1, \infty}(\Omega)$ we also have

$$
\nabla b\left(u^{n}\right) \in L^{\infty}\left(0, T ; L^{\infty}(\Omega)\right) .
$$


Next, assuming that the family of triangulations is regular, we obtain estimates uniform in $n$, from which we derive the convergence

$$
u^{n} \longrightarrow u^{\varepsilon} \quad \text { in } L^{1}\left(0, T ; L^{1}(\Omega)\right), \quad \text { as } n \rightarrow \infty .
$$

Finally, let us prove a crucial result in the context of the regularization: that the approximate solution is bounded from below away from 0 .

Lemma 3.3. It holds that

$$
u^{n}(x, t) \geq \varepsilon_{0} e^{-t}, \quad \text { for a.e. }(x, t) \in \Omega \times[0, T] .
$$

Proof. The function $v=\varepsilon_{0} e^{-t}$ satisfies the inequality

$$
\begin{aligned}
& \left(v_{t}, \phi^{n}\right)_{n}+\sum_{i=1}^{d} \int_{\Omega} a_{i}(\nabla b(v)) \partial_{i} \phi^{n}+\left(f(v), \phi^{n}\right)_{n} \\
& \quad=\left(\varepsilon_{0} e^{-t}\left(-1+\varepsilon_{0}^{k-1} e^{-(k-1) t}\right), \phi^{n}\right)_{n} \\
& \quad \leq 0
\end{aligned}
$$

for all $\phi^{n} \in S_{0}^{n}(0)$ satisfying $\phi^{n} \geq 0$. We now subtract identity (3.6) from (3.11) and integrate in time from 0 to $\tau \leq T$. Choosing

$$
\phi^{n}=\Pi_{n} g_{\delta}\left(b(v)-b\left(u^{n}\right)\right) \in S_{0}^{n}(0),
$$

where, for $0<\delta<1$,

$$
g_{\delta}(s)= \begin{cases}1 & \text { if } s>\delta \\ \delta^{-1} s & \text { if } 0 \leq s \leq \delta \\ 0 & \text { if } s<0\end{cases}
$$

we obtain

$$
\begin{aligned}
& \int_{0}^{\tau}\left(\left(v-u^{n}\right)_{t}, g_{\delta}\left(b(v)-b\left(u^{n}\right)\right)\right)_{n} \\
& \leq \quad \int_{0}^{\tau} \int_{\Omega}\left|\nabla b\left(u^{n}\right)\right|^{p-2}\left\langle\nabla\left(b\left(u^{n}\right)-b(v)\right), \nabla \Pi_{n} g_{\delta}\left(b(v)-b\left(u^{n}\right)\right)\right\rangle \\
& \quad+\int_{0}^{\tau}\left(f\left(u^{n}\right)-f(v), g_{\delta}\left(b(v)-b\left(u^{n}\right)\right)\right)_{n} .
\end{aligned}
$$

Observe that the integrals on the right-hand side are negative. In fact, $b\left(u^{n}\right)-b(v)$ is piecewise linear in $x$, so we have

$$
\begin{aligned}
& \left\langle\nabla\left(b\left(u^{n}\right)-b(v)\right), \nabla \Pi_{n} g_{\delta}\left(b(v)-b\left(u^{n}\right)\right)\right\rangle \\
& \quad=\left\langle\nabla \Pi_{n}\left(b\left(u^{n}\right)-b(v)\right), \nabla \Pi_{n} g_{\delta}\left(b(v)-b\left(u^{n}\right)\right)\right\rangle \leq 0 .
\end{aligned}
$$

Taking the $\lim _{\delta \rightarrow 0}$ and using the fact that

$$
\lim _{\delta \rightarrow 0} g_{\delta}\left(b(v)-b\left(u^{n}\right)\right)=\lim _{\delta \rightarrow 0} g_{\delta}\left(v-u^{n}\right)
$$

and $\left(v-u^{n}\right)_{t} g_{\delta}\left(v-u^{n}\right)=\partial_{t} G_{\delta}\left(v-u^{n}\right)$, where $G_{\delta}(s)=\int_{0}^{s} g_{\delta}(r) \mathrm{d} r$, we conclude that

$$
\int_{\Omega} \Pi_{n}\left\{v(\cdot, \tau)-u^{n}(\cdot, \tau)\right\}^{+} \leq \int_{\Omega} \Pi_{n}\left\{v(\cdot, 0)-u^{n}(\cdot, 0)\right\}^{+}=0 .
$$


Hence, it follows that $v\left(x_{j}, \tau\right) \leq u^{n}\left(x_{j}, \tau\right)$ for all nodes $x_{j}$ of the triangulation. This implies that $b(v) \leq b\left(u^{n}\right)$ in $\Omega \times\{\tau\}$, since $b(v)$ and $b\left(u^{n}\right)$ are piecewise linear functions. Noting that $\tau$ is arbitrary the assertion follows.

\section{Proof of the main Result}

In this section we give the proof of the smoothing property. To begin with we establish a comparison theorem (see Lemma 4.1 below).

Let $u^{n}$ be the function satisfying (3.5)-3.7. We now introduce the parabolic operator, defined by

$$
\begin{aligned}
L^{n}(v)= & v_{t}-c_{1} \frac{v}{t}-\frac{b^{\prime \prime}\left(u^{n}\right)}{t\left(b^{\prime}\left(u^{n}\right)\right)^{2}} v^{2}+f^{\prime}\left(u^{n}\right) v \\
& -b^{\prime}\left(u^{n}\right) \sum_{i} \partial_{i}\left[(p-2)\left|\nabla b\left(u^{n}\right)\right|^{p-4}\left\langle\nabla b\left(u^{n}\right), \nabla v\right\rangle \partial_{i} b\left(u^{n}\right)\right] \\
& -b^{\prime}\left(u^{n}\right) \sum_{i} \partial_{i}\left[\left|\nabla b\left(u^{n}\right)\right|^{p-2} \partial_{i} v\right],
\end{aligned}
$$

where the constant $c_{1}$ is given by

$$
c_{1}=\frac{m-1}{m(p-1)-1} \text {. }
$$

Further, we set

$$
\begin{aligned}
L^{n}\left(v, \phi^{n}\right)= & -\left(v, \phi_{t}^{n}\right)_{n}-\left(c_{1} \frac{v}{t}+\frac{b^{\prime \prime}\left(u^{n}\right)}{t\left(b^{\prime}\left(u^{n}\right)\right)^{2}} v^{2}-f^{\prime}\left(u^{n}\right) v, \phi^{n}\right)_{n} \\
& +(p-2) \int_{\Omega}\left|\nabla b\left(u^{n}\right)\right|^{p-4}\left\langle\nabla b\left(u^{n}\right), \nabla v\right\rangle\left\langle\nabla b\left(u^{n}\right), \nabla \Pi_{n}\left(b^{\prime}\left(u^{n}\right) \phi^{n}\right)\right\rangle \\
& +\int_{\Omega}\left|\nabla b\left(u^{n}\right)\right|^{p-2}\left\langle\nabla v, \nabla \Pi_{n}\left(b^{\prime}\left(u^{n}\right) \phi^{n}\right)\right\rangle-\left(v(\cdot, 0), \phi^{n}(\cdot, 0)\right)_{n}
\end{aligned}
$$

for $v \in S_{0}^{n}(\alpha)$ and $\phi^{n} \in S_{1}^{n}(0)$ with $\phi^{n}(\cdot, T)=0$. The comparison theorem reads as follows.

Lemma 4.1. Let $v \in S_{0}^{n}(\alpha), w \in S_{0}^{n}\left(\alpha^{\prime}\right), \alpha \geq \alpha^{\prime}$, and

$$
v(x, 0) \geq w(x, 0) \quad \text { in } \Omega
$$

If, for all $\phi^{n} \in S_{1}^{n}(0)$, with $\phi^{n}(\cdot, T)=0$ and $\phi^{n} \geq 0$,

$$
\int_{0}^{T} L^{n}\left(v, \phi^{n}\right) \geq \int_{0}^{T} L^{n}\left(w, \phi^{n}\right)
$$

and the integrals are well defined, then we have

$$
v(x, t) \geq w(x, t), \quad \text { for a.e. }(x, t) \in \Omega \times[0, T] .
$$


Proof. We have

$$
\begin{aligned}
0 \leq & \int_{0}^{T}\left(L^{n}\left(v, \phi^{n}\right)-L^{n}\left(w, \phi^{n}\right)\right) \\
= & -\int_{0}^{T}\left(v-w, \phi_{t}^{n}\right)_{n}-c_{1} \int_{0}^{T}\left(\frac{v-w}{t}, \phi^{n}\right)_{n} \\
& -\int_{0}^{T}\left(\frac{b^{\prime \prime}\left(u^{n}\right)}{t\left(b^{\prime}\left(u^{n}\right)\right)^{2}}\left(v^{2}-w^{2}\right), \phi^{n}\right)_{n}+\int_{0}^{T}\left(f^{\prime}\left(u^{n}\right)(v-w), \phi^{n}\right)_{n} \\
& +(p-2) \int_{0}^{T} \int_{\Omega}\left|\nabla b\left(u^{n}\right)\right|^{p-4}\left\langle\nabla b\left(u^{n}\right), \nabla(v-w)\right\rangle\left\langle\nabla b\left(u^{n}\right), \nabla \Pi_{n}\left(b^{\prime}\left(u^{n}\right) \phi^{n}\right)\right\rangle \\
& +\int_{0}^{T} \int_{\Omega}\left|\nabla b\left(u^{n}\right)\right|^{p-2}\left\langle\nabla(v-w), \nabla \Pi_{n}\left(b^{\prime}\left(u^{n}\right) \phi^{n}\right)\right\rangle \\
& -\left(v(\cdot, 0)-w(\cdot, 0), \phi^{n}(\cdot, 0)\right)_{n} \\
=: & J_{1}+\cdots+J_{7} .
\end{aligned}
$$

Now we choose an appropriate test function $\phi^{n}$. Let

$$
\psi_{\sigma}(x, t)=\frac{1}{\sigma} \int_{t}^{t+\sigma} \psi(x, \tau) d \tau
$$

and let $g_{\delta}$ be the function defined in (3.12). We set

$$
\phi^{n}(x, t)=\frac{1}{\sigma} \int_{t-\sigma}^{t} \Pi_{n} g_{\delta}\left(w_{\sigma}(x, \tau)-v_{\sigma}(x, \tau)\right)\{\tau\}^{+}\{T-\sigma-\tau\}^{+} e^{\lambda \tau} d \tau,
$$

where $\lambda<0$ is a constant, and the functions $v$ and $w$ are extended for $t<0$ and $t>T$ in an appropriate way. Let us note that $w_{\sigma}-v_{\sigma} \leq 0$ on $\partial \Omega \times(0, T]$. Thus, $\phi^{n}=0$ on $\partial \Omega \times(0, T]$. Moreover, it is not hard to see that $\phi^{n} \in S_{1}^{n}(0), \phi^{n}(\cdot, T)=0$, and $\phi^{n} \geq 0$. Hence, $\phi^{n}$ is an admissible test function.

Let us estimate the integrals $J_{1}, \ldots, J_{7}$ from above. Let

$$
D_{t}^{ \pm \sigma} f(x, t)=\frac{f(x, t \pm \sigma)-f(x, t)}{\sigma}
$$

be the difference quotients associated with $f$ and recall the definition of the function

$$
G_{\delta}(s)=\int_{0}^{s} g_{\delta}(r) \mathrm{d} r
$$

We easily see that

$$
\begin{aligned}
J_{1} & =\int_{0}^{T}\left(w-v,-D_{t}^{-\sigma}\left[g_{\delta}\left(w_{\sigma}-v_{\sigma}\right)\{t\}^{+}\{T-\sigma-t\}^{+} e^{\lambda t}\right]\right)_{n} \\
& =\int_{-\sigma}^{T-\sigma}\left(-D_{t}^{\sigma}(w-v), g_{\delta}\left(w_{\sigma}-v_{\sigma}\right)\{t\}^{+}\{T-\sigma-t\}^{+} e^{\lambda t}\right)_{n} .
\end{aligned}
$$

Due to the fact that $D_{t}^{\sigma}(w-v)=\partial_{t}\left(w_{\sigma}-v_{\sigma}\right)$, we have

$$
D_{t}^{\sigma}(w-v) g_{\delta}\left(w_{\sigma}-v_{\sigma}\right)=\partial_{t} G_{\delta}\left(w_{\sigma}-v_{\sigma}\right) ;
$$

thus, integrating by parts, we obtain

$$
J_{1}=\int_{-\sigma}^{T-\sigma}\left(G_{\delta}\left(w_{\sigma}-v_{\sigma}\right), \partial_{t}\left[\{t\}^{+}\{T-\sigma-t\}^{+} e^{\lambda t}\right]\right)_{n} .
$$


Taking the limit in $\sigma \rightarrow 0$, and noting that $\partial_{t}\left(t(T-t) e^{\lambda t}\right)=(\lambda t(T-t)+T-2 t) e^{\lambda t}$, we arrive at

$$
\lim _{\substack{\sigma \rightarrow 0 \\ \delta \rightarrow 0}} J_{1}=\lambda \int_{0}^{T}\left(\{w-v\}^{+}, t(T-t) e^{\lambda t}\right)_{n}+\int_{0}^{T}\left(\{w-v\}^{+},(T-2 t) e^{\lambda t}\right)_{n} .
$$

Next, we find

$$
\begin{aligned}
\lim _{\substack{\sigma \rightarrow 0 \\
\delta \rightarrow 0}} J_{2} & =\lim _{\delta \rightarrow 0} \int_{0}^{T}\left((w-v) g_{\delta}(w-v), c_{1}(T-t) e^{\lambda t}\right)_{n} \\
& =c_{1} \int_{0}^{T}\left(\{w-v\}^{+},(T-t) e^{\lambda t}\right)_{n}
\end{aligned}
$$

and

$$
\begin{aligned}
\lim _{\substack{\sigma \rightarrow 0 \\
\delta \rightarrow 0}} J_{3} & =\lim _{\delta \rightarrow 0} \int_{0}^{T}\left(\frac{b^{\prime \prime}\left(u^{n}\right)}{t\left(b^{\prime}\left(u^{n}\right)\right)^{2}}(v+w)(w-v) g_{\delta}(w-v), t(T-t) e^{\lambda t}\right)_{n} \\
& =\int_{0}^{T}\left(\frac{b^{\prime \prime}\left(u^{n}\right)}{\left(b^{\prime}\left(u^{n}\right)\right)^{2}}(v+w)\{w-v\}^{+},(T-t) e^{\lambda t}\right)_{n} .
\end{aligned}
$$

Noting that $f$ is a monotone increasing function we obtain

$$
\begin{aligned}
\lim _{\substack{\sigma \rightarrow 0 \\
\delta \rightarrow 0}} J_{4} & =-\lim _{\delta \rightarrow 0} \int_{0}^{T}\left(f^{\prime}\left(u^{n}\right)(w-v) g_{\delta}(w-v), t(T-t) e^{\lambda t}\right)_{n} \\
& =-\int_{0}^{T}\left(f^{\prime}\left(u^{n}\right)\{w-v\}^{+}, t(T-t) e^{\lambda t}\right)_{n} \\
& \leq 0 .
\end{aligned}
$$

Moreover $\nabla b\left(u^{n}\right), \nabla b^{\prime}\left(u^{n}\right)$, and $\nabla \Pi_{n}\left(b^{\prime}\left(u^{n}\right) g_{\delta}(w-v)\right)$ are $L^{\infty}$-functions, bounded uniform in $\delta$, so we may conclude that

$$
\lim _{\substack{\sigma \rightarrow 0 \\ \delta \rightarrow 0}}\left(J_{5}+J_{6}\right) \leq c \int_{0}^{T}\|\nabla(w-v)\|_{L^{1}(\Omega)} t(T-t) e^{\lambda t} .
$$

Thus, collecting results and noting that $J_{7} \leq 0$, we arrive at

$$
0 \leq \lim _{\substack{\sigma \rightarrow 0 \\ \delta \rightarrow 0}}\left(J_{1}+\cdots+J_{7}\right) \leq \int_{0}^{T}\left(\lambda t(T-t)\left\|\Pi_{n}\{w-v\}^{+}\right\|_{L^{1}(\Omega)}+K(t)\right) e^{\lambda t} d t
$$

where $K(t)=K_{1}(t)+K_{2}(t)$, with

$$
\begin{aligned}
K_{1}(t) & =c\left\|\Pi_{n}\left((1+v+w)\{w-v\}^{+}\right)\right\|_{L^{1}(\Omega)} \\
& \leq c\|1+v+w\|_{H^{1}(\Omega)}\|w-v\|_{H^{1}(\Omega)} \\
& \leq c\left(1+\|v\|_{H^{1}(\Omega)}^{2}+\|w\|_{H^{1}(\Omega)}^{2}\right)
\end{aligned}
$$

and

$$
K_{2}(t)=c\|\nabla(w-v)\|_{L^{1}(\Omega)} .
$$

Now let us assume that $\left\|\Pi_{n}\{w-v\}^{+}\right\|_{L^{1}(\Omega \times(0, T))}>0$. Choosing $\lambda<0$ such that $|\lambda|$ is sufficiently large we obtain a contradiction. Hence, it follows that $w\left(x_{j}, t\right) \leq$ $v\left(x_{j}, t\right)$ for all nodes $x_{j}$ of the triangulation and a.e. $t \in(0, T)$. This implies that $w \leq v$ a.e. in $\Omega \times[0, T]$, since $v$ and $w$ are piecewise linear functions. This concludes the proof. 
We now begin to state and prove a series of lemmas that will be the building blocks of the proof of our main result.

Lemma 4.2. Let $u^{n}$ be a solution of (3.5)-(3.7) and

$$
z(x, t)=\frac{m(p-2)}{m(p-1)-1} \partial_{t} b\left(u^{n}(x, t)\right) .
$$

Then

$$
\int_{0}^{T} L^{n}\left(t \partial_{t} b\left(u^{n}\right), \phi^{n}\right)=\int_{0}^{T}\left(z, \phi^{n}\right)_{n},
$$

for all $\phi^{n} \in S_{1}^{n}(0)$ such that $\phi^{n}(\cdot, T)=0$.

Proof. Let $v(x, t)=t \partial_{t} b\left(u^{n}(x, t)\right)$. Note that

$$
v(x, t)=t \partial_{t} b\left(u^{n}\right)=t b^{\prime}\left(u^{n}\right) u_{t}^{n},
$$

where $u_{t}^{n}=\partial_{t} u^{n}$. We have

$$
\begin{aligned}
J_{0} & :=\int_{0}^{T}\left(u_{t}^{n}, \partial_{t}\left(t b^{\prime}\left(u^{n}\right) \phi^{n}\right)\right)_{n} \\
& =\int_{0}^{T}\left(u_{t}^{n}, b^{\prime}\left(u^{n}\right) \phi^{n}\right)_{n}+\int_{0}^{T}\left(u_{t}^{n}, t b^{\prime \prime}\left(u^{n}\right) u_{t}^{n} \phi^{n}\right)_{n}+\int_{0}^{T}\left(u_{t}^{n}, t b^{\prime}\left(u^{n}\right) \phi_{t}^{n}\right)_{n} \\
& =: J_{1}+J_{2}+J_{3} .
\end{aligned}
$$

Let us define the constant $c_{0}=\frac{m(p-2)}{m(p-1)-1}$. Note that $1-c_{0}=\frac{m-1}{m(p-1)-1}=c_{1}$, where $c_{1}$ is the constant given in (4.1). Using equation (4.2) we get

$$
\begin{aligned}
J_{1} & =\int_{0}^{T}\left(c_{0} b^{\prime}\left(u^{n}\right) u_{t}^{n}, \phi^{n}\right)_{n}+\int_{0}^{T}\left(c_{1} b^{\prime}\left(u^{n}\right) u_{t}^{n}, \phi^{n}\right)_{n} \\
& =\int_{0}^{T}\left(z, \phi^{n}\right)_{n}+\int_{0}^{T}\left(c_{1} \frac{v}{t}, \phi^{n}\right)_{n} \\
& =: J_{11}+J_{12} .
\end{aligned}
$$

Further, due to the fact that

$$
\left(u_{t}^{n}\right)^{2}=\left(\frac{v}{t b^{\prime}\left(u^{n}\right)}\right)^{2}
$$

it follows that

$$
J_{2}=\int_{0}^{T}\left(\frac{b^{\prime \prime}\left(u^{n}\right)}{t\left(b^{\prime}\left(u^{n}\right)\right)^{2}} v^{2}, \phi^{n}\right)_{n} .
$$

Next, we have

$$
J_{3}=\int_{0}^{T}\left(v, \phi_{t}^{n}\right)_{n} .
$$

In addition, using equation (3.6), we obtain

$$
\begin{aligned}
J_{0}= & \int_{0}^{T}\left(u_{t}^{n}, \Pi_{n} \partial_{t}\left(t b^{\prime}\left(u^{n}\right) \phi^{n}\right)\right)_{n} \\
= & -\sum_{i} \int_{0}^{T} \int_{\Omega} a_{i}\left(\nabla b\left(u^{n}\right)\right) \partial_{i} \Pi_{n} \partial_{t}\left(t b^{\prime}\left(u^{n}\right) \phi^{n}\right) \\
& -\int_{0}^{T}\left(f\left(u^{n}\right), \Pi_{n} \partial_{t}\left(t b^{\prime}\left(u^{n}\right) \phi^{n}\right)\right)_{n} .
\end{aligned}
$$


Note that $u_{t}^{n}=\frac{v}{t b^{\prime}\left(u^{n}\right)}$. Thus,

$$
\partial_{t} f\left(u^{n}\right)=f^{\prime}\left(u^{n}\right) u_{t}^{n}=\frac{f^{\prime}\left(u^{n}\right) v}{t b^{\prime}\left(u^{n}\right)} .
$$

Integration by parts yields

$$
\begin{aligned}
-\int_{0}^{T}\left(f\left(u^{n}\right), \Pi_{n} \partial_{t}\left(t b^{\prime}\left(u^{n}\right) \phi^{n}\right)\right)_{n} & =-\int_{0}^{T}\left(f\left(u^{n}\right), \partial_{t}\left(t b^{\prime}\left(u^{n}\right) \phi^{n}\right)\right)_{n} \\
& =\int_{0}^{T}\left(f^{\prime}\left(u^{n}\right) v, \phi^{n}\right)_{n} .
\end{aligned}
$$

Moreover, we find

$$
\partial_{t} b\left(u^{n}\right)=b^{\prime}\left(u^{n}\right) u_{t}^{n}=\frac{v}{t}
$$

and

$$
\begin{aligned}
\partial_{t} a_{i}\left(\nabla b\left(u^{n}\right)\right)= & (p-2)\left|\nabla b\left(u^{n}\right)\right|^{p-4}\left\langle\nabla b\left(u^{n}\right), \nabla \partial_{t} b\left(u^{n}\right)\right\rangle \partial_{i} b\left(u^{n}\right) \\
& +\left|\nabla b\left(u^{n}\right)\right|^{p-2} \partial_{i} \partial_{t} b\left(u^{n}\right) \\
= & (p-2)\left|\nabla b\left(u^{n}\right)\right|^{p-4}\left\langle\nabla b\left(u^{n}\right), \nabla \frac{v}{t}\right\rangle \partial_{i} b\left(u^{n}\right) \\
& +\left|\nabla b\left(u^{n}\right)\right|^{p-2} \partial_{i} \frac{v}{t}
\end{aligned}
$$

Thus, we obtain

$$
\begin{aligned}
J_{0}= & \int_{\Omega}(p-2)\left|\nabla b\left(u^{n}\right)\right|^{p-4}\left\langle\nabla b\left(u^{n}\right), \nabla v\right\rangle\left\langle\nabla b\left(u^{n}\right), \nabla \Pi_{n}\left(b^{\prime}\left(u^{n}\right) \phi^{n}\right)\right\rangle \\
& +\int_{\Omega}\left|\nabla b\left(u^{n}\right)\right|^{p-2}\left\langle\nabla v, \nabla \Pi_{n}\left(b^{\prime}\left(u^{n}\right) \phi^{n}\right)\right\rangle+\int_{0}^{T}\left(f^{\prime}\left(u^{n}\right) v, \phi^{n}\right)_{n} .
\end{aligned}
$$

Altogether, it follows that

$$
\int_{0}^{T} L^{n}\left(v, \phi^{n}\right)=-J_{3}-J_{12}-J_{2}+J_{0}=J_{11}=\int_{0}^{T}\left(z, \phi^{n}\right)_{n}
$$

for all $\phi^{n} \in S_{1}^{n}(0)$ with $\phi^{n}(\cdot, T)=0$. Thus the assertion is proven.

Lemma 4.3. Let $c_{2}=\frac{m}{m(p-1)-1}$ and $z(x, t)=\frac{m(p-2)}{m(p-1)-1} \partial_{t} b\left(u^{n}(x, t)\right)$. Then

$$
\int_{0}^{T} L^{n}\left(-c_{2} b\left(u^{n}\right), \phi^{n}\right) \leq \int_{0}^{T}\left(z, \phi^{n}\right)_{n}
$$

for all $\phi^{n} \in S_{1}^{n}(0)$ such that $\phi^{n}(\cdot, T)=0$ and $\phi^{n} \geq 0$. 
Proof. Let us compute $L^{n}\left(-c_{2} b\left(u^{n}\right), \phi^{n}\right)$. We have

$$
\begin{aligned}
\int_{0}^{T} & L^{n}\left(-c_{2} b\left(u^{n}\right), \phi^{n}\right) \\
= & \int_{0}^{T}\left(c_{2} b\left(u^{n}\right), \phi_{t}^{n}\right)_{n}+\int_{0}^{T}\left(c_{1} \frac{c_{2} b\left(u^{n}\right)}{t}, \phi^{n}\right)_{n} \\
& -\int_{0}^{T}\left(\frac{b^{\prime \prime}\left(u^{n}\right)}{t\left(b^{\prime}\left(u^{n}\right)\right)^{2}}\left(c_{2} b\left(u^{n}\right)\right)^{2}, \phi^{n}\right)_{n}-\int_{0}^{T}\left(f^{\prime}\left(u^{n}\right) c_{2} b\left(u^{n}\right), \phi^{n}\right)_{n} \\
& -(p-2) \int_{0}^{T} \int_{\Omega}\left|\nabla b\left(u^{n}\right)\right|^{p-4}\left\langle\nabla b\left(u^{n}\right), c_{2} \nabla b\left(u^{n}\right)\right\rangle\left\langle\nabla b\left(u^{n}\right), \nabla \Pi_{n}\left(b^{\prime}\left(u^{n}\right) \phi^{n}\right)\right\rangle \\
& -\int_{0}^{T} \int_{\Omega}\left|\nabla b\left(u^{n}\right)\right|^{p-2}\left\langle c_{2} \nabla b\left(u^{n}\right), \nabla \Pi_{n}\left(b^{\prime}\left(u^{n}\right) \phi^{n}\right)\right\rangle+\left(c_{2} b\left(u_{0}^{n}\right), \phi^{n}(\cdot, 0)\right)_{n} \\
=: & J_{1}+\cdots+J_{7} .
\end{aligned}
$$

Let us estimate $J_{1}+J_{4}+J_{5}+J_{6}+J_{7}$. We find

$$
J_{1}+J_{7}=-c_{2} \int_{0}^{T}\left(u_{t}^{n}, \Pi_{n}\left(b^{\prime}\left(u^{n}\right) \phi^{n}\right)\right)_{n} .
$$

In view of the fact that $k \geq m(p-1)$ and $u^{n}>0$ we get

$$
\begin{aligned}
J_{4} & =-c_{2} \int_{0}^{T}\left(f^{\prime}\left(u^{n}\right) b\left(u^{n}\right), \phi^{n}\right)_{n} \\
& =-\frac{c_{2} k}{m} \int_{0}^{T}\left(f\left(u^{n}\right) b^{\prime}\left(u^{n}\right), \phi^{n}\right)_{n} \\
& \leq-c_{2}(p-1) \int_{0}^{T}\left(f\left(u^{n}\right), \Pi_{n}\left(b^{\prime}\left(u^{n}\right) \phi^{n}\right)\right)_{n} .
\end{aligned}
$$

Further, let us note that

$$
J_{5}=-c_{2}(p-2) \int_{0}^{T} \int_{\Omega} \sum_{i} a_{i}\left(\nabla b\left(u^{n}\right)\right) \partial_{i} \Pi_{n}\left(b^{\prime}\left(u^{n}\right) \phi^{n}\right)
$$

and

$$
J_{6}=-c_{2} \int_{0}^{T} \int_{\Omega} \sum_{i} a_{i}\left(\nabla b\left(u^{n}\right)\right) \partial_{i} \Pi_{n}\left(b^{\prime}\left(u^{n}\right) \phi^{n}\right) .
$$

Using equation (3.6) it follows that

$$
(p-1)\left(J_{1}+J_{7}\right)+J_{4}+J_{5}+J_{6} \leq 0 ;
$$

thus,

$$
\begin{aligned}
J_{1}+J_{4}+J_{5}+J_{6}+J_{7} & \leq(1-(p-1))\left(J_{1}+J_{7}\right) \\
& =(p-2) c_{2} \int_{0}^{T}\left(b^{\prime}\left(u^{n}\right) u_{t}^{n}, \phi^{n}\right)_{n}=\int_{0}^{T}\left(z, \phi^{n}\right)_{n} .
\end{aligned}
$$

Next, let us show that $J_{2}+J_{3}=0$. We have

$$
\begin{aligned}
J_{2}+J_{3} & =\int_{0}^{T}\left(\frac{c_{1} c_{2} b\left(u^{n}\right)}{t}, \phi^{n}\right)_{n}-\int_{0}^{T}\left(\frac{c_{2}^{2} b^{\prime \prime}\left(u^{n}\right)\left(b\left(u^{n}\right)\right)^{2}}{t\left(b^{\prime}\left(u^{n}\right)\right)^{2}}, \phi^{n}\right)_{n} \\
& =\int_{0}^{T}\left(\frac{c_{2}^{2} b\left(u^{n}\right)}{t}\left(\frac{c_{1}}{c_{2}}-\frac{b^{\prime \prime}\left(u^{n}\right) b\left(u^{n}\right)}{\left(b^{\prime}\left(u^{n}\right)\right)^{2}}\right), \phi^{n}\right)_{n} .
\end{aligned}
$$


Further, it holds that

$$
\frac{c_{1}}{c_{2}}=\frac{m-1}{m}
$$

and

Thus, we obtain

$$
\frac{b^{\prime \prime}\left(u^{n}\right) b\left(u^{n}\right)}{\left(b^{\prime}\left(u^{n}\right)\right)^{2}}=\frac{m-1}{m} .
$$

$$
J_{2}+J_{3}=0
$$

Altogether, the result follows.

Lemma 4.4. Let $u^{n}$ be a solution of (3.5)-(3.7). The pointwise estimate

$$
u_{t}^{n} \geq-\frac{c}{t} u^{n}
$$

holds for a.e. $t>0$, where $c=\frac{1}{m(p-1)-1}$.

Proof. Lemma 4.2 and Lemma 4.3 yield

$$
\int_{0}^{T} \int_{\Omega} L^{n}\left(t \partial_{t} b\left(u^{n}\right), \phi^{n}\right) \geq \int_{0}^{T} \int_{\Omega} L^{n}\left(-c_{2} b\left(u^{n}\right), \phi^{n}\right),
$$

for all $\phi^{n} \in S_{1}^{n}(0)$, with $\phi^{n}(\cdot, T)=0$ and $\phi^{n} \geq 0$, where $c_{2}=\frac{m}{m(p-1)-1}>0$. Let us note that

$$
t \partial_{t} b\left(u^{n}\right)=0 \quad \text { for } t=0
$$

and

$$
-c_{2} b\left(u_{0}^{n}\right)<0
$$

Further, we have

$$
t \partial_{t} b\left(u^{n}\right)=0 \quad \text { on } \partial \Omega \times(0, T]
$$

and

$$
-c_{2} b\left(u^{n}\right)<0 \quad \text { on } \partial \Omega \times(0, T] .
$$

Thus, applying the comparison theorem (Lemma 4.1) we obtain

$$
t \partial_{t} b\left(u^{n}\right) \geq \frac{-m}{m(p-1)-1} b\left(u^{n}\right) .
$$

This implies that

$$
u_{t}^{n}=\frac{b^{\prime}\left(u^{n}\right) u_{t}^{n}}{b^{\prime}\left(u^{n}\right)} \geq \frac{-m}{[m(p-1)-1] t} \frac{b\left(u^{n}\right)}{b^{\prime}\left(u^{n}\right)}=\frac{-1}{[m(p-1)-1] t} u^{n},
$$

since $b^{\prime}\left(u^{n}\right)>0$ and $b^{\prime}\left(u^{n}\right) u^{n}=m\left|u^{n}\right|^{m-1} u^{n}=m b\left(u^{n}\right)$.

We conclude the paper with the proofs of Theorem 2.5 and Corollary 2.6.

Proof of Theorem 2.5. We multiply the pointwise estimate (4.3) by a smooth function $0 \leq \varphi \in \mathcal{D}(\Omega \times(0, T))$ and integrate by parts in time. Due to the fact that $u^{n} \rightarrow u^{\varepsilon}$ and $u^{\varepsilon} \rightarrow u$ in $L^{1}(\Omega \times(0, T))$, we obtain

$$
\int_{0}^{T} \int_{\Omega} u \varphi_{t} \leq \int_{0}^{T} \int_{\Omega} \frac{c}{t} u \varphi, \quad \forall \varphi \in \mathcal{D}(\Omega \times(0, T)): \varphi \geq 0,
$$

where $c=\frac{1}{m(p-1)-1}$. 
Proof of Corollary 2.6. We multiply equation (3.1) by $\phi=g_{\delta}\left(b\left(u^{\varepsilon}\right)-\varepsilon^{m}\right)$, where $g_{\delta}$ is defined in (3.12), and integrate in time from 0 to $\tau$. Noting that $\nabla g_{\delta}\left(b\left(u^{\varepsilon}\right)-\varepsilon^{m}\right)=$ $g_{\delta}^{\prime}\left(b\left(u^{\varepsilon}\right)-\varepsilon^{m}\right) \nabla b\left(u^{\varepsilon}\right), g_{\delta}^{\prime} \geq 0$, and $f\left(u^{\varepsilon}\right) g_{\delta}\left(b\left(u^{\varepsilon}\right)-\varepsilon^{m}\right) \geq 0$ we find

$$
\int_{0}^{\tau} \int_{\Omega} u_{t}^{\varepsilon} g_{\delta}\left(b\left(u^{\varepsilon}\right)-\varepsilon^{m}\right) \leq 0 .
$$

Using the fact that $\lim _{\delta \rightarrow 0} g_{\delta}\left(b\left(u^{\varepsilon}\right)-b(\varepsilon)\right)=\lim _{\delta \rightarrow 0} g_{\delta}\left(u^{\varepsilon}-\varepsilon\right)$ and $u_{t}^{\varepsilon}=\left(u^{\varepsilon}-\varepsilon\right)_{t}$ we obtain

$$
\int_{\Omega}\left\{u^{\varepsilon}(\cdot, \tau)-\varepsilon\right\}^{+} \leq \int_{\Omega}\left\{u_{0}^{\varepsilon}-\varepsilon\right\}^{+} .
$$

This yields

$$
\int_{\Omega}\left\{u^{\varepsilon}(\cdot, \tau)\right\}^{+} \leq \int_{\Omega}\left\{u_{0}^{\varepsilon}\right\}^{+}+c \varepsilon
$$

that is,

$$
\left\|u^{\varepsilon}(\cdot, \tau)\right\|_{L^{1}(\Omega)} \leq\left\|u_{0}^{\varepsilon}\right\|_{L^{1}(\Omega)}+c \varepsilon, \quad \text { a.e. } \tau>0 .
$$

Taking the limit $\lim _{\varepsilon \rightarrow 0}$ and noting that spt $u$ is bounded, we conclude that

$$
\|u(\cdot, \tau)\|_{L^{1}\left(\mathbb{R}^{d}\right)} \leq\left\|u_{0}\right\|_{L^{1}\left(\mathbb{R}^{d}\right)}, \quad \text { a.e. } \tau>0 .
$$

The proof of the corollary now follows from this and Theorem 2.5] using standard arguments (cf. [14]).

\section{REFERENCES}

1. D.G. Aronson and Ph. Bénilan, Régularité des solutions de l'équation des milieux poreux dans $\mathbb{R}^{n}$, C.R. Acad. Sci. Paris Sér. A-B 288 (1979), no. 2, A103-A105. MR0524760 (82i:35090)

2. G.I. Barenblatt, On self-similar motions of compressible fluids in porous media, Prik. Math Mech. 16 (1952), 679-698. (Russian) MR0052948(14:699h)

3. F. Bernis, Existence results for doubly nonlinear higher order parabolic equations on unbounded domains, Math. Ann. 279 (1988), 373-394. MR0922422 (89j:35062)

4. D. Blanchard and G. Francfort, Study of a doubly nonlinear heat equation with no growth assumption on the parabolic term, SIAM J. Math. Anal 19 (1988), 1032-1056. MR0957665 (90e:35087a)

5. L. A. Caffarelli and A. Friedman, Regularity of the free boundary of a gas flow in an n-dimensional porous medium, Indiana Univ. Math. J. 29 (1980), 361-391. MR0570687(82a:35096)

6. M.G. Crandall and M. Pierre, Regularization effects for $u_{t}+A \varphi(u)=0$ in $L^{1}$, J. Funct. Anal. 45 (1982), no. 2, 194-212. MR0647071 (83g:34071)

7. M.G. Crandall and M. Pierre, Regularization effects for $u_{t}+\Delta \varphi(u)=0$, Trans. Amer. Math. Soc. 274 (1982), no. 1, 159-168. MR0670925 (84h:35081)

8. J.L. Diaz and J.F. Padial, Uniqueness and existence of solutions in the $B V_{t}(Q)$ space to a doubly nonlinear parabolic problem, Publ. Math. Barc. 40 (1996), 527-560. MR1425634 (97j:35078)

9. E. DiBenedetto, Degenerate Parabolic Equations, New York, Springer-Verlag, 1993. MR1230384 (94h:35130)

10. E. DiBenedetto, J.M. Urbano and V. Vespri, Current issues on singular and degenerate evolution equations, in: Handbook of Differential Equations, Evolutionary Equations, volume 1 (C.M. Dafermos and E. Feireisl eds.), Elsevier B.V., 2004.

11. C. Ebmeyer, Error estimates for a class of degenerate parabolic equations, SIAM J. Num. Anal. 35 (1998), 1095-1112. MR1619863 (99e:65125)

12. C. Ebmeyer, A non-degeneracy property for a class of degenerate parabolic equations, Zeit. Anal. Anwend. 15 (1996), 637-650. MR1406080 (97e:35096)

13. C. Ebmeyer and J.M. Urbano, Regularity in Sobolev spaces for doubly nonlinear parabolic equations, J. Differential Equations 187 (2003), no. 2, 375-390. MR1949446 (2003m:35113)

14. J.R. Esteban and J.L. Vazquez, Homogeneous diffusion in $\mathbb{R}$ with power-like nonlinear diffusivity, Arch. Rat. Mech. Anal. 103 (1988), 39-80. MF0946969 (89j:35064) 
15. J.R. Esteban and J.L. Vazquez, Régularité des solutions positives de l'équation parabolique p-Laplacienne, C. R. Acad. Sci. Paris Sér I 310 (1990), 105-110. MR.1044625 (91i:35036)

16. J. Filo, Local existence and $L^{\infty}$-estimate of weak solutions to a nonlinear degenerate parabolic equation with nonlinear boundary data, Panam. Math. J. 4 (1994), no. 3, 1-31. MR1290044 (95h:35120)

17. M.A. Herrero and J.L. Vazquez, The one-dimensional nonlinear heat equation with absorption: regularity of solutions and interfaces, SIAM J. Math. Anal 18 (1987), 149-167. MR0871827 (88a:35124)

18. A.V. Ivanov, Existence and uniqueness of a regular solution of the Cauchy-Dirichlet problem for doubly nonlinear parabolic equations, Zeit. Anal. Anwend. 14 (1995), 751-777. MR1376576 (96m:35173)

19. A.V. Ivanov, Regularity for doubly nonlinear parabolic equations, J. Math. Sci. 83 (1997), no. 1, 22-37. MR.1328634 (96b:35096)

20. J.L. Lions, Quelques méthodes de résolution des problèmes aux limites non linéaires, Paris, Dunod, 1969. MR0259693 (41:4326)

21. F. Otto, $L^{1}$-contraction and uniqueness for quasilinear elliptic-parabolic equations, J. Differential Equations 131 (1996), 20-38. MR 1415045|(97i:35125)

22. M.M. Porzio and V. Vespri, Hölder estimates for local solutions of some doubly nonlinear degenerate parabolic equations, J. Differential Equations 103 (1993), 146-178. MR1218742 (94d:35015)

23. F. Simondon, Effet régularisant local pour $u_{t}=(\phi(u))_{x x}$, C. R. Acad. Sci. Paris Sér I 299 (1984), 969-972. MR0774680 (86j:35094)

24. J.M. Urbano, Hölder continuity of local weak solutions for parabolic equations exhibiting two degeneracies, Adv. Differential Equations 6 (2001), no. 3, 327-358. MR1799489 (2001j:35173)

25. J.M. Urbano, Continuous solutions for a degenerate free boundary problem, Ann. Mat. Pura Appl. (IV) 178 (2000), 195-224. MF 1849386 (2002h:35353)

26. J.L. Vazquez and M. Wallas, Existence and uniqueness of solutions of diffusion-absorption equations with general data, Differ. Integral Equations 7 (1994), 15-36. MR1250936 (94k:35170)

27. V. Vespri, On the local behaviour of solutions of a certain class of doubly nonlinear parabolic problems, Manuscripta Math. 75 (1992), no. 1, 65-80. MR.1156216 (93d:35071)

Mathematisches Seminar, Universität Bonn, Nussallee 15, D-53115 Bonn, Germany

E-mail address: cebmeyer@uni-bonn.de

Departamento de Matemática, Universidade de Coimbra, 3001-454 Coimbra, Portugal

E-mail address: jmurb@mat.uc.pt 